\title{
Fluoroscopy integrating technology in a 3D mapping system during ablation of atrial arrhythmias: first experiences
}

\author{
Christian Blockhaus, Jan Schmidt, Muhammed Kurt, Lukas Clasen, Patrick Müller, \\ Christoph Brinkmeyer, Malte Kelm, Dong-In Shin, Hisaki Makimoto
}

Division of Cardiology, Pulmonology, and Vascular Medicine, Heinrich Heine University, Medical Center Düsseldorf, Düsseldorf, Germany

Submitted: 6 November 2015

Accepted: 15 June 2016

Arch Med Sci 2018; 14, 4: 794-800

DOI: https://doi.org/10.5114/aoms.2016.61944

Copyright (c) 2016 Termedia \& Banach

\section{Abstract}

Introduction: Ablation of the cavotricuspid isthmus (CTI) in patients with atrial flutter (AFL) and pulmonary vein isolation (PVI) in patients with atrial fibrillation (AF) are both common therapies. As the demand for ablative treatments rises, total radiation exposure times of staff increase concomitantly. Here, we report on our first experiences with a new fluoroscopy integrating system (FIS) integrated into a current 3D mapping system (3DMS).

Material and methods: The study population consisted of 59 consecutive patients who underwent PVI or CTI ablation (26 and 33 patients with and without FIS respectively). Total procedure time (PT), fluoroscopy exposure time (FT) and dose-area product (DAP) were monitored.

Results: All procedures were successfully completed without major complications. Employing FIS in the PVI group, FT and DAP were both significantly reduced after completing a short learning curve of 6 cases (respectively $361.6 \pm 181$ s vs. $530.3 \pm 156.7$ s, $p=0.039 ; 801.9 \pm 439.15$ cGycm $^{2}$ vs. 1495 $\pm 435.2 \mathrm{cGycm}^{2}, p=0.002$ ). Mean PT was not significantly affected (121 \pm 26.7 min vs. $135.6 \pm 23.2 \mathrm{~min}, p=0.21$ ). The same holds true for CTI ablation: FT $(99.29 \pm 51.4$ s vs. $153.9 \pm 76.6 \mathrm{~s}, p=0.022)$ and DAP $(269 \pm 128.7$ $\mathrm{cGycm}^{2}$ vs. $524.3 \pm 288.4$ cGycm $^{2}, p=0.002$ ) were significantly reduced, leaving PT not significantly affected ( $29.5 \pm 10 \mathrm{~min}$ vs. $35.2 \pm 16.3 \mathrm{~min}, p=0.23)$. Conclusions: The introduction of the new FIS with a current 3DMS results in a significant reduction of both the total FT and DAP without affecting PT. The initial learning curve for adopting this method is considerably short.

Key words: atrial fibrillation, atrial flutter, radiation, ablation, electroanatomical mapping.

\section{Introduction}

Prevalence of atrial fibrillation (AF) is rising due to the growth of the elderly population [1]. After the development of catheter ablation therapy as a cornerstone therapy, the number of pulmonary vein isolations $(\mathrm{PVI})$ is also increasing, which leads on average to higher exposure to radiation both for physicians and patients [2].

This increasing radiation may result in severe complications in the long run. Several studies have described the influence of radiation in the development of malignancies [3, 4]. They showed for example acute

\author{
Corresponding author: \\ Univ. Prof. Dr. \\ Hisaki Makimoto \\ Division of Cardiology, \\ Pulmonology, and \\ Vascular Medicine \\ Heinrich Heine \\ University \\ Medical Center \\ Moorenstr. 5 \\ 40225 Düsseldorf, Germany \\ Phone: +49 2118118800 \\ E-mail: hisaki.makimoto@ \\ med.uni-duesseldorf.de
}


DNA damage in patients undergoing radiation but also discussed the development of brain tumors in interventional cardiologists.

Three-dimensional mapping systems (3DMS) allowing the investigator to visualize the catheter on a screen are widely used in ablation of arrhythmias and are already leading to a reduction of fluoroscopy. However, efforts should still be undertaken to use as little radiation as possible.

A new fluoroscopy integrating system (FIS), CARTO UNIVU module (Biosense Webster, Diamond Bar, CA, USA), used with the 3DMS CARTO3 (Biosense Webster, Diamond Bar, CA, USA) has been introduced recently. It enables the integration of fluoroscopic cine loops and images into the 3DMS at various angles. This technique has been described in detail recently [5]. Briefly, the CARTO location pad is equipped with a registration pad and the CARTO system is upgraded with the UNIVU module software. After a quick registration the module allows the real-time visualization of intracardiac catheters against a background of stored fluoroscopic images or cines. Several fluoroscopic images are acquired at different angulations and stored. After being transferred, the image is integrated into the 3D map, allowing the user to create an electro-anatomical map on top of the captured fluoroscopic image or cine.

Here, we report our first experiences with this new module during PVI procedures and ablations of cavotricuspid isthmi (CTI) as atrial flutter (AFL) treatment with a commercially available irrigated ablation catheter.

\section{Material and methods}

The study population consisted of 59 patients who underwent catheter ablation of AF or AFL. Prospective use of the FIS with 3DMS was achieved consecutively in 16 patients with AF and 17 with common AFL. Ten control PVI cases and 16 cases with ablation of common AFL were consecutively enrolled retrospectively before the introduction of the FIS. Patients with prior left atrial (LA) ablation were excluded. All patients provided written informed consent for the procedure.

Total procedure time (PT) (skin to skin), fluoroscopic time (FT) and dose-area product (DAP) data were collected. All images were taken with 3.75 frames per second. All PT, FT, and DAP were included in the analysis to evaluate the value of FIS in real clinical practice. In a more detailed analysis we distinguished three working steps during the procedure. During PVI these steps were: puncture to begin LA mapping, LA map construction, and finally ablation. During ablation of AFL the steps were: puncture until catheter placement, mapping of CTI, and ablation.

All patients undergoing PVI were anticoagulated for at least 4 weeks prior to the procedure. Phen- procoumon was not stopped before the procedure. Administration of direct oral anticoagulants was ceased 1 day before the procedure. All patients underwent transesophageal echocardiography to exclude thrombus within the left atrial appendage.

The procedure was performed under deep sedation using midazolam and continuous infusion of propofol. Detailed information about the ablation procedure before introduction of the FIS has been published previously [6]. In short, a 6-F catheter was placed inside the coronary sinus (CS) via the right femoral vein and, after transseptal punctures (TSP), two SL1 sheaths (St Jude Medical, St Paul, $M N$ ) were advanced into the LA. After TSP, activated clotting time (ACT) was monitored every $15 \mathrm{~min}$ and heparin was used to maintain ACT > $300 \mathrm{~s}$. Selective angiography of the PVs was performed in the right anterior oblique (RAO) $30^{\circ}$ and left anterior oblique (LAO) $40^{\circ}$ view to define the PV ostia. For ablation an irrigated quadripolar catheter (Thermocool SmartTouch, Biosense Webster) was used. We relied on contact-force measurements, which have been proven to show a beneficial effect on the results of the PVI procedure [7].

Circumferential PVI was performed with $30 \mathrm{~W}$ (17 $\mathrm{ml} / \mathrm{min}$ flow) on the anterior wall and $25 \mathrm{~W}$ (17 $\mathrm{ml} / \mathrm{min}$ flow) on the posterior wall. We used a multipolar diagnostic catheter (Lasso, Biosense Webster) for mapping and proving both entry and exit block. All PVI procedures were performed by the same experienced physician.

As for the ablation of AFL, one octapolar catheter was placed in the right ventricular apex. Mapping of the CTI was undertaken with the same kind of irrigated quadripolar ablation catheter as used for the PVIs.

The FIS images were taken in AP (anterior-posterior), RAO $30^{\circ}$, and $\mathrm{LAO} 60^{\circ}$ at the beginning of LA mapping or before CTI mapping. During PVI, the cine-loops of selective PV angiography (RAO $30^{\circ}$ and LAO $40^{\circ}$ ) were also taken into the CARTO system.

The endpoint of the PVI procedure was defined as bidirectional block (entrance and exit block), proven by the absence of any PV spike recorded on the Lasso catheter when placed within the ipsilateral PVs at least 20 min after PVI and by the absence of LA capture under pacing from the Lasso catheter placed within the ipsilateral PVs $(5 \mathrm{mV}$, 1 ms output). For CTI ablation the endpoint was defined as a bidirectional block proven by a decreasing delay of time after stimulation from the catheter on CTI next to the ablation line and subsequently more lateral during pacing from both the CS and the mapping catheter at 500 ms intervals.

All authors had full access to the data, and have read and agreed to the manuscript as written. The study was approved by the local Institutional Review Board. 


\section{Statistical analysis}

For global test statistics we used a significance level of $5 \%$. Continuous data are shown as mean \pm standard deviation (SD). The $\chi^{2}$ test, Student's $t$ test, or 1-way analysis of variance was performed when appropriate. The analyses of our data were performed using SPSS (IBM SPSS Statistics, Version 22.0, Armonk, NY, USA).

\section{Results}

Between 10/2014 and 09/2015, 16 PVIs and 17 CTI ablations were performed with FIS, and 10 PVIs and $16 \mathrm{CTI}$ ablations were undertaken without FIS.

In all cases isolation of all four PVs or a bidirectional CTI block was achieved respectively. There were no major complications or adverse effects such as pericardial effusion, stroke or hemorrhage in our cohort.

The baseline characteristics of the two groups did not differ concerning age, sex, comorbidities, LA diameter, ejection fraction (EF) or medication (Tables I and II). In the group undergoing CTI ablation without FIS, patients had significantly more coronary artery disease and a slightly higher body mass index (BMI).

At the beginning of FIS introduction we could observe a learning curve of only six procedures before a decrease of FT and DAP was apparent. Figure 1 shows a decrease of these values with stable PT, while, in contrast, all PVIs without FIS showed stable values concerning PT, FT and DAP (Figure 2). For further analysis we excluded the first 6 PVIs, representing the learning curve.

Table I. Baseline characteristics of CTI patients

\begin{tabular}{|lccc|}
\hline Parameter & FIS (+) & FIS (-) & $P$-value \\
\hline Gender (male) & $8(47)$ & $10(62.5)$ & 0.37 \\
\hline Age [years] & $65.24 \pm 13.84$ & $66.44 \pm 13.23$ & 0.8 \\
\hline Ejection fraction (\%) & $60.41 \pm 5.9$ & $57.62 \pm 7.57$ & 0.25 \\
\hline Left atrial diameter $[\mathrm{mm}]$ & $39.94 \pm 7.11$ & $36.62 \pm 9.51$ & 0.26 \\
\hline Body mass index $\left[\mathrm{kg} / \mathrm{m}^{2}\right]$ & $24.89 \pm 4.95$ & $28.16 \pm 4.78$ & 0.06 \\
\hline Coronary artery disease & $2(11.8)$ & $7(43.8)$ & 0.04 \\
\hline Cardiac surgery & $8(47)$ & $3(18.75)$ & 0.08 \\
\hline Peripheral arterial disease & $1(5.9)$ & $0(0)$ & 0.32 \\
\hline Arterial hypertension & $14(82.3)$ & $10(62.5)$ & 0.2 \\
\hline Diabetes mellitus II & $2(11.8)$ & $2(12.5)$ & 0.95 \\
\hline Dyslipidemia & $11(61)$ & $8(50)$ & 0.39 \\
\hline Stroke & $3(17.65)$ & $1(6.25)$ & 0.32 \\
\hline Atrial fibrillation & $14(88.6)$ & $12(75)$ & 0.61 \\
\hline Creatinine $[\mathrm{mg} / \mathrm{dl}]$ & $0.99 \pm 0.22$ & $1.03 \pm 0.27$ & 0.62 \\
\hline
\end{tabular}

All values $n(\%)$ or mean $\pm S D$.

Table II. Baseline characteristics of PVI patients

\begin{tabular}{|lccc|}
\hline Parameter & FIS (+) & FIS (-) & $P$-value \\
\hline Sex (male) & $7(43.75)$ & $3(30)$ & 0.48 \\
\hline Age [years] & $67.8 \pm 9.9$ & $60 \pm 11.7$ & 0.085 \\
\hline Ejection fraction (\%) & $57 \pm 12.8$ & $57.7 \pm 4.7$ & 0.87 \\
\hline Left atrial diameter [mm] & $40.37 \pm 7.16$ & $38.2 \pm 7.8$ & 0.47 \\
\hline Body mass index & $26.87 \pm 5.6$ & $28.63 \pm 3.94$ & 0.4 \\
\hline Coronary artery disease & $4(25)$ & $3(30)$ & 0.78 \\
\hline Cardiac surgery & $3(18.75)$ & $2(20)$ & 0.94 \\
\hline Peripheral arterial disease & $1(6.25)$ & $0(0)$ & 0.42 \\
\hline Arterial hypertension & $13(81.25)$ & $5(50)$ & 0.09 \\
\hline Diabetes mellitus II & $4(18)$ & $1(10)$ & 0.34 \\
\hline Dyslipidemia & $9(40.6)$ & $4(40)$ & 0.42 \\
\hline Stroke & $4(18)$ & $0(0)$ & 0.086 \\
\hline Creatinine [mg/dl] & $1.19 \pm 0.72$ & $0.94 \pm 0.22$ & 0.31 \\
\hline
\end{tabular}

All values $n(\%)$ or mean $\pm S D$. 


\section{The CTI ablation}

As previously described, we analyzed in detail the data concerning the different steps during CTI ablation in 16 patients without FIS and 17 patients with FIS.

As shown in Figure $3 \mathrm{~A}$, the PT was not affected significantly from puncture to catheter placement $(13.8 \pm 5.5$ min with FIS vs. $13.7 \pm 7.4$ min without FIS, $p=0.95)$ or during CTI mapping (6.2 $\pm 5 \mathrm{~min}$ with FIS vs. $4.6 \pm 2.2 \mathrm{~min}$ without FIS, $p=0.26$ ). The duration from the beginning of radiofrequency (RF) application to the end of the procedure was significantly reduced $(9.5 \pm 4.5 \mathrm{~min}$ with FIS vs. $16.9 \pm 12.7$ min without FIS, $p=0.03$ ). Total PT was not affected significantly $(29.5 \pm 10 \mathrm{~min}$ with FIS vs. $35.2 \pm 16.3$ min without FIS, $p=0.23$ ).

As shown in Figure $3 \mathrm{~B}$, the FT was significantly reduced after initialization of the FIS module after catheter placement (puncture until start of CTI mapping: $82.8 \pm 48.8 \mathrm{~s}$ with FIS vs. $100.2 \pm 55.6 \mathrm{~s}$ without FIS, $p=0.34$; CTI-map: $11.9 \pm 8.4 \mathrm{~s}$ with FIS vs. $25.6 \pm 18.8$ s without FIS, $p=0.01$; ablation:

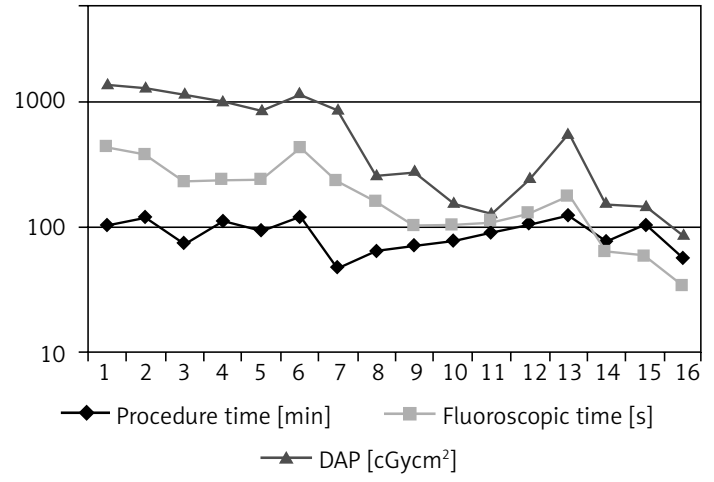

Figure 1. Learning curve with FIS after transseptal puncture (TSP). After approximately 6 patients FT and DAP showed a decrease although the procedure time was not affected (logarithmic scale)

A

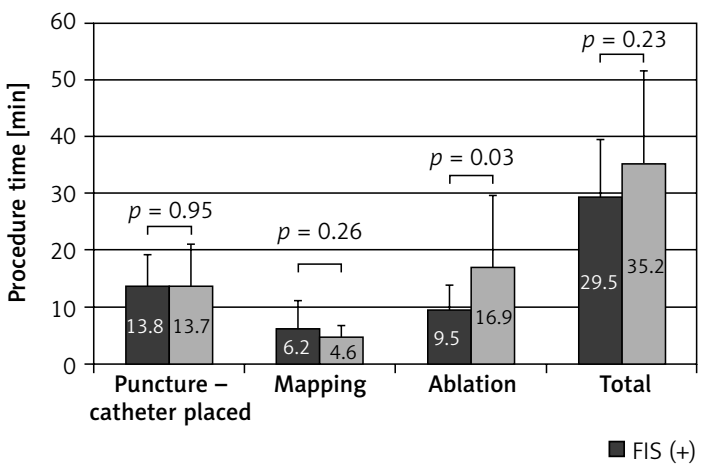

$5.2 \pm 7.9$ s with FIS vs. $28.1 \pm 26.9$ s without FIS, $p=$ 0.002 ; total: $99.3 \pm 51.4 \mathrm{~s}$ with FIS vs. $153.9 \pm 76.6 \mathrm{~s}$ without FIS, $p=0.022$ ). The DAP was also reduced significantly after catheter placement (puncture until start of CTI mapping: $218.1 \pm 117.9 \mathrm{cGycm}^{2}$ with FIS vs. $329 \pm 1933$ cGycm $^{2}$ without FIS, $p=0.054$; CTI-map: $35.5 \pm 27.6$ cGycm $^{2}$ with FIS vs. $92 \pm 78.5$ cGycm $^{2}$ without FIS, $p=0.009$; ablation: $15.5 \pm 24.4$ cGycm $^{2}$ with FIS vs. $103.3 \pm 116.5$ cGycm $^{2}$ without FIS, $p=0.005$; total: $269 \pm 128.7 \mathrm{cGycm}^{2}$ with FIS vs. $524.3 \pm 288.4 \mathrm{cGycm}^{2}$ without FIS, $p=0.002$ ).

Concerning the follow-up results of $\mathrm{CTI}$ ablation we observed only one patient with recurrent AFL in the group without FIS and no recurrence in the group using FIS $(p=0.3)$. The mean follow-up period was $207 \pm 114$ days and data were available for all patients.

\section{The PVI}

As previously described, we analyzed the data concerning the different steps during PVI in 10 patients without FIS and 10 patients with FIS.

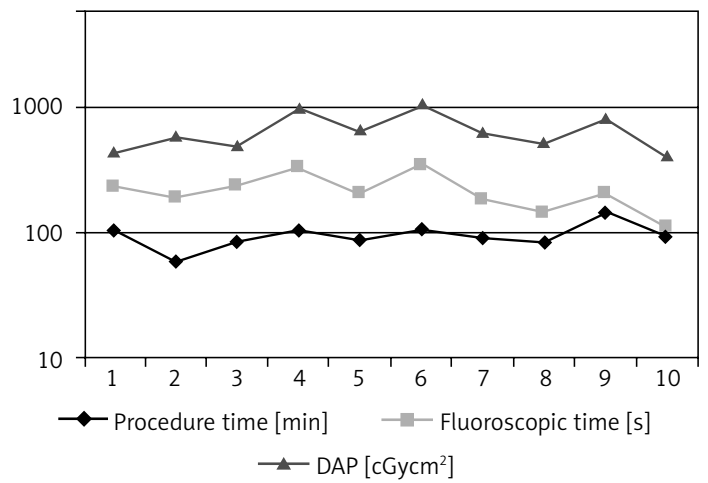

Figure 2. Stable curve without FIS after TSP. During these prospective cases, no serial change was observed in PT, FT or DAP during PVI without FIS (logarithmic scale)

B

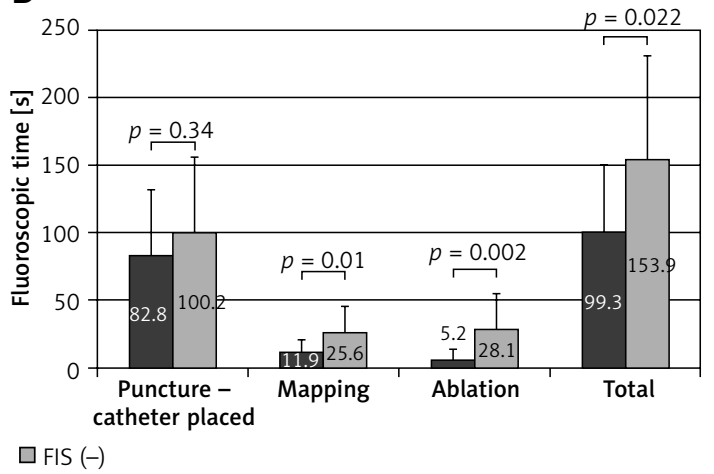

Figure 3. Comparison of procedure time (PT), fluoroscopic time (FT), and dose-area product (DAP) with and without fluoroscopic integrating system (FIS) during ablation of atrial flutter (AFL). A - The use of FIS did not affect the PT as compared to the procedure without FIS in each procedure phase. B - The use of FIS reduced the FT significantly as compared to the procedure without FIS. Significance was observed only for the time after the initialization of FIS. Before starting catheter mapping there was no significant difference in FT 
A

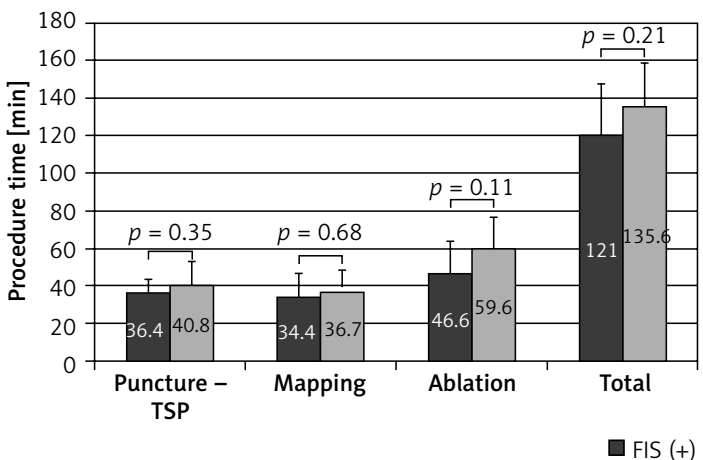

B

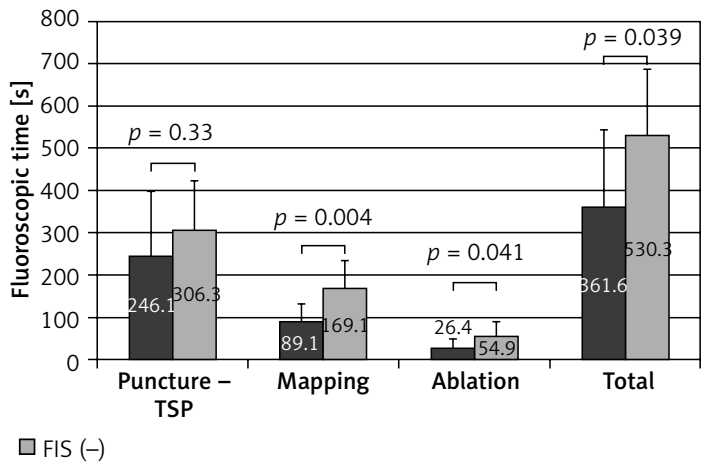

Figure 4. Comparison of PT, FT, and DAP with and without FIS during PVI. A - The PT was not significantly affected by the use of FIS in each phase during PVI. B - The FT was significantly reduced by the use of FIS. Significance was observed only for the time after the initialization of FIS

As shown in Figure 4 A, the PT was not affected significantly either during the time from puncture to start of LA mapping (36.4 \pm 7 min with FIS vs. $40.8 \pm 12.7$ min without FIS, $p=0.35$ ) or during LA mapping ( $34.4 \pm 12.7$ min with FIS vs. $36.7 \pm 11.7$ min without FIS, $p=0.68$ ) or during ablation (46.6 \pm 17.4 min with FIS vs. $59.6 \pm 16.9$ min without FIS, $p=0.11)$. Total PT was $121 \pm 26.7$ min with FIS vs. $135.6 \pm 23.2$ min without FIS $(p=0.21)$.

As shown in Figure 4 B, the FT was significantly reduced after initialization of the FIS module after TSP (puncture until start of LA mapping: 246.1 \pm 151.7 s with FIS vs. $306.3 \pm 116$ s without FIS $p=0.33$; LA-map: $89.1 \pm 43.5$ s with FIS vs. 169.1 \pm 64.4 s without FIS, $p=0.004$; ablation: 26.4 \pm 22.2 s with FIS vs. $54.9 \pm 34.5$ s without FIS, $p=0.041$; total: $361.6 \pm 181$ s with FIS vs. 530.3 $\pm 156.7 \mathrm{~s}$ without FIS, $p=0.039$ ). Also, DAP was reduced significantly after TSP (puncture until start of LA mapping: $518.4 \pm 278.7 \mathrm{cGycm}^{2}$ with FIS vs. $830 \pm 399.8$ cGycm $^{2}$ without FIS, $p=0.06$; LA-map: $221.9 \pm 180.5$ cGycm $^{2}$ with FIS vs. $508.9 \pm 214.5$ $\mathrm{cGycm}^{2}$ without FIS, $p=0.005$; ablation: $61.7 \pm 70.6$ $\mathrm{cGycm}^{2}$ with FIS vs. $156 \pm 84.5 \mathrm{cGycm}^{2}$ without FIS, $p=0.014$; total: $801.9 \pm 439.2 \mathrm{cGycm}^{2}$ with FIS vs. $1495 \pm 435.2 \mathrm{cGycm}^{2}$ without FIS, $p=0.002$ ).

Follow-up was available for all patients. After a mean follow-up period of $208 \pm 92$ days, assum-

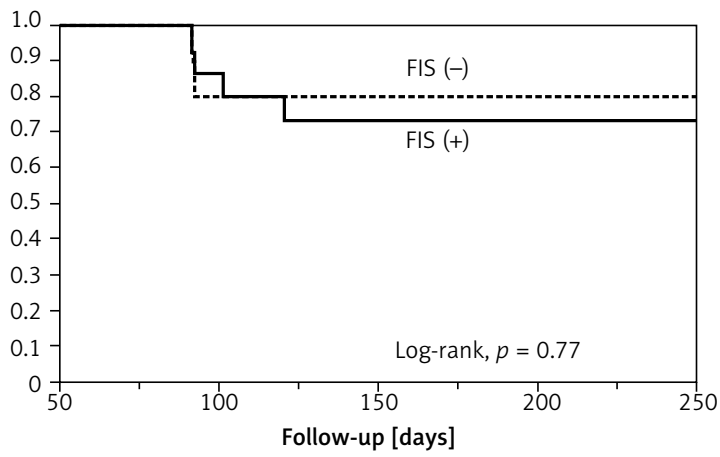

Figure 5. Follow-up data for patients undergoing PVI. No significant outcome was observed ing a blanking period of 3 months, we found that $75 \%$ of patients were free from any recurrence of AF or any other arrhythmia in the FIS group and $80 \%$ in the group without FIS ( $p=0.77$, Figure 5).

\section{Discussion}

Our study shows that the integration of the FIS module into the 3DMS allows a significant decrease in FT and DAP without affecting the PT and without leading to additional adverse effects during either PVI or CTI ablation. It also demonstrates a relatively short learning curve to implement use of this module into the standard procedure. To the best of our knowledge, this is the first report on such a short learning curve.

The PVI is widely used as a cornerstone therapy for patients with symptomatic AF. However, those patients may have recurrent $A F$ and atrial tachycardias after PVI depending on background heart diseases, gene mutations, and life styles [1, 8]. While the need for atrial arrhythmia ablation is increasing, it is even more necessary to protect patients and staff from radiation. Exposure to radiation has been identified as a risk for chromosomal damage and the development of tumors in patients and in interventional cardiologists [3, 4 , 9]. Several studies have been published with attempts to reduce radiation during daily work $[2,10]$. Heidbuchel et al. reported that an effective dose of $15 \mathrm{mSv}$ (millisieverts) is associated with an excess cancer risk of 1 in 750 men aged 50 years. The risk is 38\% higher in females, 3-4 fold higher in children and one-half higher in the elderly [2]. In this work the total DAP in PVIs was reduced from an average of $1495 \mathrm{cGycm}^{2}$ to $801 \mathrm{cGycm}^{2}$. This difference of $694 \mathrm{cGycm}^{2}$ is equivalent to approximately $1.38 \mathrm{mSv}$.

The introduction of 3DMSs (CARTO3, Biosense Webster and NAVX, St. Jude Medical), used in the ablation of mainly complex arrhythmias, has already led to a decrease in radiation exposure, as it allows 
the physician to move catheters in reconstructed 3D maps. Their safety and benefit have been widely demonstrated $[11,12]$. One limiting factor is the lack of possibility to integrate fluoroscopic cines and loops, which appears especially useful in obtaining LA anatomy and the position of ostia of PVs. Using the FIS module, investigators' ability to precisely identify vessels, understand the anatomy of the heart, interpret angiographic cines and move catheters more confidently can be strengthened.

With the FIS module the integration of fluoroscopic loops and pictures in different angles with the 3D anatomical map enables the confirmation of catheter position without any additional fluoroscopy (Figure 6), resulting in an even greater decrease of radiation. Also, selective PV angiography was undertaken independently of the use of FIS.

Not only for atrial arrhythmias, for which we have demonstrated FIS' usefulness in the present study, but also for other ablation procedures such as ventricular tachycardias, it might prove to be advantageous in further reducing fluoroscopy, for example due to the integration of additionally performed angiography of coronary arteries or the coronary sinus, thus providing valuable surplus information.

In the present study, FIS was only initialized after TSP or catheter placement, which explains the confinement of reduction of radiation to all steps after TSP or after catheter placement (Figure 4 B). This accords with recently published works by other groups $[5,13,14]$.

During ablation we used LAO $40^{\circ}$ and RAO $30^{\circ}$ projections. It is known that the $\mathrm{LAO}$ projection leads to a higher DAP, especially in obese patients, than the RAO projection. An ablation protocol preferring more RAO projections could also lead to a reduction of DAP [10].

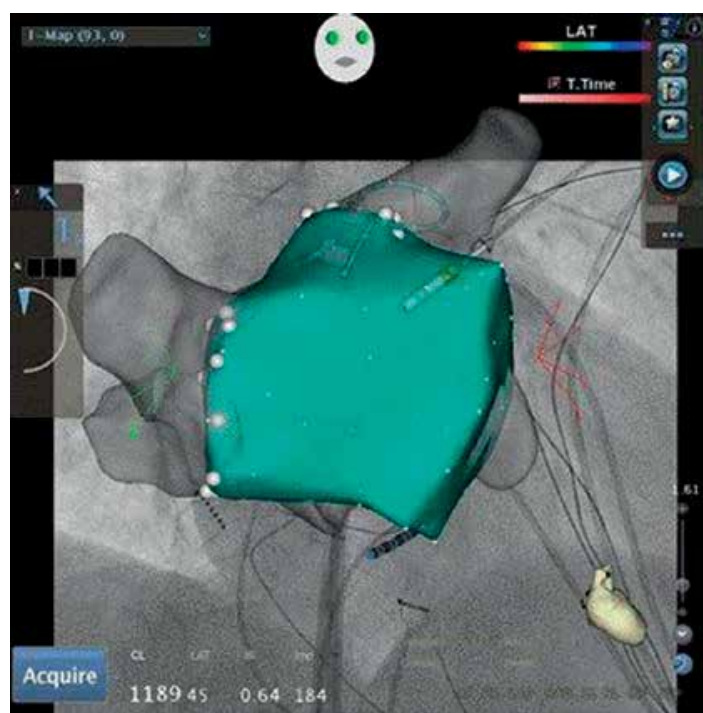

Recently, another radiation reducing system comparable to the FIS used in this work has been introduced (MEDIGuide, St. Jude Medical). It has been shown to be a capable tool which significantly decreases FT and DAP but with remarkable asset costs [15-17].

The finding of the present study that neither the total PT nor the time of all substeps was affected by the use of the FIS suggested that using FIS does not influence the process of PVI or CTI ablation. The significant reduction of fluoroscopy even during a relatively simple and short mapping time for CTI underscores the efficacy of FIS.

Another aim to achieve would be "zero" fluoroscopy ablation. Several studies have reported on this, but still safety and feasibility have to be proven in further studies [11, 18-21].

Eickholt et al. reported a case of real-time contact force guided nonfluoroscopic ablation [22]. This FIS works effectively only after introducing the mapping and ablation catheter into the heart. This means that the FIS usefulness is exerted only after TSP during PVI and after placing catheters in the right heart during $\mathrm{CTI}$ ablation. As we have shown in Figure $3 \mathrm{~B}$ and $4 \mathrm{~B}$, fluoroscopy was mostly necessary before these steps. More options to reduce the need of radiation before these steps, particularly TSP, would be desirable. With respect to this, intracardiac ultrasound and/or RF transseptal needle should be mentioned [23] as further potentially helpful tools.

This was a single-center and retrospective study. However, to demonstrate its efficacy only a small number of procedures were necessary. Therefore we believe that the usefulness of this FIS can be emphasized even more. We did not observe any complication during the ablation. This

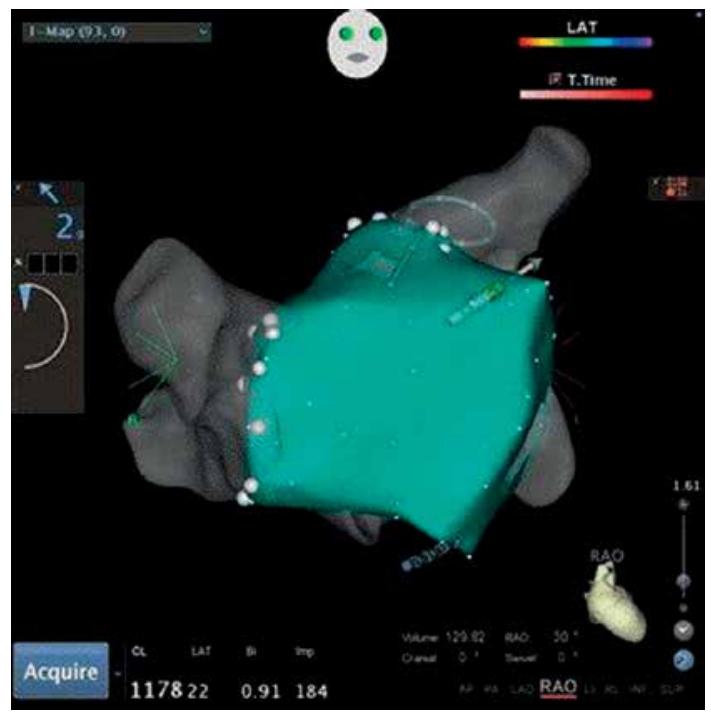

Figure 6. RAO view of the left atrium with (left side) and without (right side) FIS. With FIS (left panel) it is also possible to run cinematic loops of $P V$ angiography. Therefore once the cinematic loops were taken, the necessity for additional use of fluoroscopy during LA mapping and ablation can be significantly diminished 
may be based on the small number of patients not allowing us to draw a conclusion. There are no long-term follow-up data available yet. Also we do not expect any differences in the two groups. In this study we did not adopt the fluoroscopic aperture. This implementation should lead to further DAP reduction. Finally, for medical staff in the catheter laboratory a radiation monitoring system may be essential to evaluate the precise radiation dose and spatial variability, which was not possible in the present study. Implementation of apparatus such as the special cabin system which Narbutt et al. recently reported should be evaluated in future studies [24].

In conclusion, a new FIS module integrated into the existing 3D mapping system significantly reduces the exposure of patients and staff to radiation during $\mathrm{PVI}$ and ablation of the common AFL without affecting the catheter ablation procedures. This fluoroscopy reduction is possible only after a considerably short learning curve.

\section{Conflict of interest}

The authors declare no conflict of interest.

\section{References}

1. Chugh SS, Havmoeller R, Narayanan K, et al. Worldwide epidemiology of atrial fibrillation: a Global Burden of Disease 2010 Study. Circulation 2014; 129: 837-47.

2. Heidbuchel H, Wittkampf F, Vano E, et al. Practical ways to reduce radiation dose for patients and staff during device implantations and electrophysiological procedures. Europace 2014; 16: 946-64.

3. Roguin A, Goldstein J, Bar O. Brain tumors among interventional cardiologists: a cause for alarm? Report of four new cases from two cities and a review of the literature. Eurolntervention 2012; 7: 1081-6.

4. Andreassi MG, Cioppa A, Manfredi S, Palmieri C, Botto N, Picano E. Acute chromosomal DNA damage in human lymphocytes after radiation exposure in invasive cardiovascular procedures. Eur Heart J 2007; 28: 2195-9.

5. Akbulak RÖ, Schäffer B, Jularic M, et al. Reduction of radiation exposure in atrial fibrillation ablation using a new image integration module: a prospective randomized trial in patients undergoing pulmonary vein isolation. J Cardiovasc Electrophysiol 2015; 26: 747-53.

6. Makimoto $\mathrm{H}$, Heeger $\mathrm{CH}$, Lin $\mathrm{T}$, et al. Comparison of contact force-guided procedure with non-contact force-guided procedure during left atrial mapping and pulmonary vein isolation: impact of contact force on recurrence of atrial fibrillation. Clin Res Cardiol 2015; 104: 861-70.

7. Wutzler A, Huemer M, Parwani AS, Blaschke F, Haverkamp W, Boldt LH. Contact force mapping during catheter ablation for atrial fibrillation: procedural data and one-year follow-up. Arch Med Sci 2014; 10: 266-72.

8. Kiliszek M, Kozluk E, Franaszczyk M, et al. The 4q25, $1 \mathrm{q} 21$, and $16 \mathrm{q} 22$ polymorphisms and recurrence of atrial fibrillation after pulmonary vein isolation. Arch Med Sci 2016; 12: 38-44.

9. Pearce MS, Salotti JA, Little MP, et al. Radiation exposure from CT scans in childhood and subsequent risk of leukaemia and brain tumours: a retrospective cohort study. Lancet 2012; 380: 499-505.

10. Nof E, Lane C, Cazalas M, et al. Reducing radiation exposure in the electrophysiology laboratory: it is more than just fluoroscopy times! Pacing Clin Electrophysiol 2015; 38: 136-45.

11. Casella M, Pelargonio G, Dello Russo A, et al. "Near-zero" fluoroscopic exposure in supraventricular arrhythmia ablation using the EnSite NavX ${ }^{\mathrm{TM}}$ mapping system: personal experience and review of the literature. J Interv Card Electrophysiol 2011; 31: 109-18.

12. Stabile G, Scaglione M, del Greco M, et al. Reduced fluoroscopy exposure during ablation of atrial fibrillation using a novel electroanatomical navigation system: a multicentre experience. Europace 2012; 14: 60-5.

13. Christoph M, Wunderlich C, Moebius S, et al. Fluoroscopy integrated 3D mapping significantly reduces radiation exposure during ablation for a wide spectrum of cardiac arrhythmias. Europace 2015; 17: 928-37.

14. Cano Ó, Alonso P, Osca J, et al. Initial experience with a new image integration module designed for reducing radiation exposure during electrophysiological ablation procedures. J Cardiovasc Electrophysiol 2015; 26: 662-70.

15. Sommer P, Richter S, Hindricks G, Rolf S. Non-fluoroscopic catheter visualization using MediGuide ${ }^{\mathrm{TM}}$ technology: experience from the first 600 procedures. J Interv Card Electrophysiol 2014; 40: 209-1

16. Sommer P, Piorkowski C, Gaspar T, et al. MediGuide in supraventricular tachycardia: initial experience from a multicentre registry. Europace 2013; 15: 1292-7.

17. Muser D, Magnani S, Santangeli P. Ablation of typical atrial flutter using the novel MediGuide 3D catheter tracking system: a review of the literature. Expert Rev Cardiovasc Ther 2014; 12: 799-802.

18. Kerst G, Weig HJ, Weretka S, et al. Contact force-controlled zero-fluoroscopy catheter ablation of right-sided and left atrial arrhythmia substrates. Heart Rhythm 2012; 9: 709-14.

19. Bulava A, Hanis J, Eisenberger M. Catheter ablation of atrial fibrillation using zero-fluoroscopy technique: a randomized trial. Pacing Clin Electrophysiol 2015; 38: 797-806.

20. Koźluk E, Gawrysiak M, Piątkowska A, et al. Radiofrequency ablation without the use of fluoroscopy - in what kind of patients is it feasible? Arch Med Sci 2013; 9: 821-5.

21. Anselmino M, Sillano D, Casolati D, Ferraris F, Scaglione M, Gaita F. A new electrophysiology era: zero fluoroscopy. J Cardiovasc Med 2013; 14: 221-7.

22. Eickholt C, Boring YC, Kelm M, Shin DI, Meyer C. Nonfluoroscopic catheter ablation of a double-loop re-entry tachycardia guided by real-time contact force information. Can J Cardiol 2013; 29: 254.e9-254.e11.

23. Hsu JC, Badhwar N, Gerstenfeld EP, et al. Randomized trial of conventional transseptal needle versus radiofrequency energy needle puncture for left atrial access (the TRAVERSE-LA study). J Am Heart Assoc 2013; 2: e000428.

24. Narbutt J, Pawlaczyk M, Sysa-Jędrzejowska A, et al. System formonitoring UV radiation level in phototherapy cabins in Poland. Arch Med Sci 2014; 10: 1244-54. 